\title{
LEPRA REACTION AND THE GENERAL ADAPTATION SYNDROME
}

\author{
By E. Muir, M.D., F.R.C.S.E., C.M.G., C.I.E.
}

\section{Introduction}

Lepra reaction, in its various forms and symptoms, is one of the most distressing complications of leprosy. Little is known about its direct cause, but it is generally agreed that a reliable method of prevention or control would considerably simplify and shorten the period of treatment, save much suffering, and remove an important cause of deformity.

The objects of this paper are: (1) without dogmatizing to suggest a hypothesis which seems to the writer to harmonize to a certain extent with the principal known facts about lepra reaction, and (2) to indicate lines of investigation which might gather evidence in favour of or against that hypothesis. A list of suggested objects for further study is given at the end of the paper.

The General Adaptation Syndrome, as set forth by Professor Hans SElye, in his book on Stress*, seems to throw light on some of the problems connected with leprosy, and particularly on the nature and treatment of lepra reaction $†$ in its two forms: erythema nodosum leprosum‡ (ENL) and acute exacerbation (AE).

ENL is recognised by the sudden appearance of raised, erythematous patches or nodules on the skin of patients with the lepromatous or dimorphous form of leprosy. These patches or nodules generally appear on the sites of already visible skin lesions, but may become visible on apparently healthy skin. Histologically they show oedema, dilatation of cutaneous vessels, infiltration with lymphocytes and mononuclear phagocytes. There is also permeation of polymorphs in direct proportion to the severity of the reaction.

ENL can occur spontaneously without an apparent cause, but it is most commonly associated with chemotherapy and the administration of sulphones, particularly when these are given in excess of the tolerance of the patient and without careful grading of initial dosage. When sulphone therapy is the cause, temporary suspension of the

* Selye, H. (1955) Stress, Montreal.

$\dagger$ It is acknowledged that there are valid objections to the term "lepra reaction" since all the manifestations of leprosy are reactions to Myco. leprae. But I have employed it for want of a more suitable generic term, and because it is in general use and is generally understood.

$\ddagger$ Erythema nodosum leprosum, as originally described by M. Murata (Lep. Rev. April 1958, 116) obviously included both forms of lepra reaction. But in recent years it has generally been restricted to the milder form of reaction, chiefly though not exclusively associated with chemotherapy. Some writers consider the term inappropriate as the lesion in leprosy does not show identical pathological features with classical erythema nodosum, but it is used here as the term generally applied and understood. 
drug or lowered dosage generally results in the disappearance of the inflammatory signs, but sometimes ENL continues or passes on into AE.

$\mathrm{AE}$ is similar in nature to $\mathrm{ENL}$, but much more severe in degree. It affects not only the skin but also the buccal and respiratory mucous membranes, the peripheral nerves, the eyes, and in fact any or all of the regions where lepra bacilli are present. Biopsy shows severe oedema, infiltration and destruction of tissue, with large numbers of polymorphs present. Nodules may suppurate and discharge pus laden with lepra bacilli. There is rise of temperature with general febrile symptoms, and there may be considerable pain and swelling in affected peripheral nerves. There is less tendency than in ENL towards self-healing, and a greater inclination towards repeated relapse. ENL may be aggravated into $\mathrm{AE}$ if the cause is not removed in time.

\section{The General Adaptation Syndrome (GAS)}

This is described by SELYE as the manifestation of "the sum of all specific systemic reactions of the body which ensue upon longcontinued exposure to systemic stress." He divides the syndrome into three stages:

1. The Alarm Reaction: This may be called forth by 'alarming stimuli' or 'stressors' and lasts until adaptation has begun to take place. The Alarm Reaction has two phases: shock and countershock. During shock there are such manifestations as hypothermia, hypotension, blood concentration, deranged capillary and cellmembrane permeability, and generalised tissue breakdown. There is a discharge of adrenaline, corticotrophin and corticoids, which are early defense reactions. During the phase of counter-shock the phenomena of defense develop. There is enlargement of the adrenal cortex, with signs of increased activity. The phase of counter-shock passes into the second stage, that of resistance.

2. The Stage of Resistance: According to SELYE this "represents the sum of all the non-specific reactions elicited by prolonged exposure to stimuli to which the organism has acquired adaptation. It is mainly characterised by an increased resistance to the particular stress or agent to which the body has been exposed, and a decreased resistance to other stimuli. Thus the impression is gained that during the stage of Resistance adaptation to one agent is acquired at the expense of resistance to other agents", whereas during the Alarm Reaction Stage there is loss of lipids from the adrenal cortex, there is deposition of lipids into this gland during the Stage of Resistance.

3. The Stage of Exhaustion "represents the sum of all nonspecific systemic reactions which ultimately develops as a result of prolonged over-exposure to stimuli to which adaptation has been developed, but could no longer be maintained". 


\section{GAS and Specific Adaptive Reactions}

"The GAS must be distinguished from the specific adaptive reactions, such as the hypertrophy of the musculature after prolonged exercise, the allergic or immunologic phenomena elicited by certain foreign proteins or microorganisms, etc. These latter responses are not evoked by systemic stress. They usually endow the body with a great deal of resistance against one particular agent (that to which it had previously been exposed), but both the manifestations of such specific adaptive reactions and the resistance which they confer upon the organism are limited to the agent which elicited them".

It has been held by some leprologists that lepra reaction (ENL and $\mathrm{AE}$ ) is of an allergic nature, but two facts suggest that it is more related to GAS, as described above, than to allergy. These two facts are: (1) lepra reaction appears not as a uniform response to one agent, but as a response to a large number of widely differing agents; (2) it appears most commonly (as will be shown below) in subjects with low general resistance.

The tissue changes described under the Alarm Reaction of GAS (blood concentration, deranged capillary and cell-membrane permeability, and generalised tissue breakdown) correspond with those found in lepra reaction, and it seems reasonable to expect that these changes should be most readily produced and most marked in tissues already weakened and damaged by leprosy infection.

\section{Stressor Agents}

SELYE mentions a large number of stressor agents whose manifestations are "so preponderantly due to abnormal adaptive reactions that the direct consequences of the eliciting pathogenic agent assume a secondary position". He compares the convulsions of tetanus toxin in which abnormal adaptive processes are relatively unimportant, with such diseases as hypertension and gastric ulcer in which "the abnormal adaptive response to the agent is the major cause of disease". This is especially obvious since "the same pathogenic stimulus, which produces such changes in one person, elicits no significant disturbances in others whose adaptive mechanism functions normally".

From among the many stressor agents and groups of stressor agents discussed by SELYE I have selected four as having particular relevance to leprosy and lepra reaction. These are (1) sulphur derivative, (2) muscular exercise (excessive or deficient), (3) diet (deficient, excessive or unbalanced), and (4) complicating diseases. It will be noticeable that in all of these power of adaptation is closely connected with the endocrine system, and more particularly with the efficient functioning or failure of the adrenal cortex.

1. Sulphur Derivatives. SEL YE writes that considerable attention has been given in recent years to adrenal changes caused by various 
organic sulphur derivatives, and especially by the thioureas. "It was found in the rat that addition of $1 \%$ thiourea to drinking water produced atrophy, simultaneously with signs of hypothyroidism. After 3 or 4 months of treatment with thiouracil all 3 layers of the adrenal cortex showed considerably involution with lipid deposition in the fasciculata; occasionally there were haemorrhages and cysts in the reticularis. Simultaneously there is a progressive decrease in the size of the adrenal medulla. The cortical involution may be regarded in a large measure as an extreme attempt of compensation, probably due to depression of the adrenocorticotrophic factor, since the cortex of such animals promptly responds to infections with hypertrophy. "The production of adreno-cortical atrophy with thioureas has been repeatedly confirmed in the rat, cat, mouse and guinea-pig. It can be counteracted by simultaneous thyroxine administration and, since this atrophy is essentially similar to that produced by thyroidectomy, it is probably due to the resulting hypothyroidism. In the rat occasionally the cortical involution is sufficiently severe to produce a functional hypocorticoidism in which $\mathrm{NaCl}$ or cortical extract treatment appear to be beneficial.

"In patients with fatal sulphathiazole intoxication the adrenals often show foci within the inner fasciculata and reticularis, the medulla is apparently not involved.

"Experimental observations suggest that certain sulphur-containing compounds such as sulphonal or sulphocyanate are concentrated in the adrenal cortex following oral administration, especially when free elimination is prevented".

I a m not aware whether or not the effect of sulphone administration on the adrenal cortex of experimental animals has been studied but, if the effects are similar to those described above as produced by other sulphur compounds, this would suggest an explanation of lepra reaction in both its $E N L$ and $A E$ forms.

It is a well known clinical experience in sulphone therapy of leprosy that patients are intolerant of full doses at the beginning of treatment. The most common sign of intolerance is lepra reaction, in either its ENL or AE form. Tolerance can however generally be induced by grading the dosage over a period of weeks or months till the patient can stand the most effective amounts.

On the supposition that sulphone acts as a 'stressor agent', this gradual acquisition of tolerance appears to correspond with the General Adaptation Syndrome. Each successive 'alarm-reaction' dose is followed by a higher Stage of Resistance until the patient becomes inured to the most beneficial dosage.

The GAS may also explain the difference between ENL and AE. If sulphone is pressed still further beyond the tolerance of the patient, AE results. This would correspond with the Stage of Exhaustion in which, as mentioned above, "adaptation to 
prolonged over-exposure to stimuli can no longer be maintained".

We are justified in considering that this Stage of Exhaustion resulting in $\mathrm{AE}$ is caused by hypocorticoidaemia, consequent on the failure of the adrenal cortex to function normally, since $A E$ is promptly relieved by oral administration of corticoids.

2. Muscular Exercise and Inactivity. SELYE states that intense muscular exercise in rats, especially in those sensitised by fasting, elicits loss of chromaffin granules of the adrenal, and occasionally extensive necrosis of medullary tissue, as well as cortical enlargement with lipid discharge. He says that his investigations "revealed that in the rat the adrenal becomes extremely hyperaemic during the first hours of muscular exercise, that is at a time corresponding to the Alarm Reaction." Experiments on guinea-pigs showed that, as a result of long-continued training, both the number of cortical sinusoids and their blood content increase, especially in the fasciculate region. Those vascular changes are also similar to those observed upon exposure to alarming stimuli. "Numerous experimental observations in various animals show that sudden intense muscular fatigue causes depletion in cortical sudanophil lipids, while during subsequent rest an inverse reaction takes place". He mentions that a similar depletion of cortical lipids was often reported in prolonged motor agitation due to various diseases. He quotes one investigator as reporting an absolute loss of adrenal weight in rats exposed to brief anorexia, and concluding that this is probably due to loss of cortex tissue, since the water content is actually increased, and histological examination reveals areas of necrosis, exhaustion, atrophy and oedema, Selye remarks that "since cytolytic phenomena are quite characteristic of very intense alarm-reactions, these observations are not in contradiction with the generally accepted view that the response of the adrenal to anorexia is essentially the same as to any other stressor".

Later, SELYE writes: "The changes accompanying severe muscular fatigue have been ascribed to a relative corticoid deficiency comparable to that produced by infections, irradiations, trauma, etc. This is in accord with our concept of derangements due to hypoadaptation. In some respects, however, the systemic consequence of exercise differ from those produced by other alarming stimuli. Exercise produces remarkably little shock and very pronounced counter-shock phenomena. There is an especially intense and prolonged hyperchloraemia, and, after an initial fall in blood sugar, there follows a marked hyperglycaemia".

SELYE also mentions that the wild Norway rat has much larger adrenals than the inbred albino laboratory rat, and that this is mainly a difference in the development of the cortex. He suggests that more frequent exposure to stress in the case of the wild rat may be respon- 
sible for this difference, interpreting the phenomenon in terms of the General Adaptation Syndrome.

In contrast with muscular exercise, SELYE describes the effects of excessive rest. "Bed-rest alone can cause marked losses of nitrogen and potassium, a decrease in blood volume, and considerable vasomotor disturbances. The resemblance of these manifestations to those seen in various spontaneous diseases has been emphasised and the delay of recovery from disease caused by extensive 'therapeutic' bed-rest has been illustrated by many examples. Lack of exercise itself requires adaptive adjustments and, since it represents a pronounced deviation from normalcy, it may perhaps act as an 'alarming stimulus'. The possibility has not yet been adequately studied, but observations on the rat suggest that forced restraint can produce General Adaptation Syndrome changes".

There is thus evidence that both excessive muscular exercise, and excessive rest, act as stressor agents, and that at least the former and probably also the latter may help to lead the subject to a state of exhaustion in which adaptation can no longer be maintained, this stage coinciding with damage to and exhaustion of the adrenals.

On the other hand, if stressors are applied moderately in the form of muscular exercise graded according to the tolerance of the subject, resistance can be built up enabling the subject to tolerate not only more strenuous muscular exercise but also other stressors which may offer challenge. Of all stressors muscular exercise is the most suitable for building up general resistance because, as mentioned above, exercise produces remarkably little shock and very pronounced counter-shock phenomena. I shall attempt to show below how muscular exercise in the form of physical training is important in the treatment of leprosy, and particularly in the prevention and cure of lepra reaction.

3. Deficient, Excessive and Unbalanced Diet. Reference has been made above to the effects on the adrenals when rats sensitized by fasting are subjected to intense muscular exercise. SELYE states that "various types of unbalanced diets, which cause stress, have been shown to produce typical GAS changes in the adrenals of animals and man. The total fat content of the cortex increases while the birefringent lipid granules tend to disappear from the cortex during prolonged starvation in birds. This is accompanied by an increase in the size of the cortex". Also in the fasting dog the adrenal cholesterol falls below normal. "In the guinea-pig the principal changes observed during starvation are: hypertrophy of the cortex with an increase in total fat and a decrease in birefringent lipid and ascorbic acid content". There are also similar changes in the mouse, rabbit and rat. "Perusal of the literature suggests that complete acute starvation acts as an alarming stimulus and causes an increase in cortical size with loss of lipids, while chronic underfeeding can elicit a stage of resis- 
tance with an increase in the lipid content and size of the cortex, presumably due to inhibition of ACTH production. Thus, if the composition of the diet is kept in mind, it appears that partial starvation acts like any other stressor in that it elicits ACTH discharge, but the effects of this response can be modified by conditioning factors". SELYE also mentions that "in man observations concerning the effects of hunger on the adrenals have often been made on a large scale in times of famine. Most investigators agree that the lipid content of the cortical cells can remain high even after most of the fat disappeared from other tissues. Only in very grave acute starvation is there considerable hydropic degeneration, haemorrhage and cytolysis in the adrenal cortex. All these data are consonant with the view that in man, as in animals, chronic undernutrition tends to cause resistant stage manifestations with lipid storage in the adrenlas, while acute complete starvation imitates the alarm reaction with a predominance of cytolytic phenomena and lipid discharge".

Overfeeding likewise causes adrenal enlargement, for instance in the rat. Such an enlargement is produced not only by acute 'food shock' but also by chronic overfeeding. "The possibility has been considered that this again may merely be a manifestation of the GAS. Presumably adaptation both to lack and to excess of food elicits GAS changes in the adrenal".

Various qualitatively inadequate diets cause GAS responses in the suprarenals. To quote SELYE: "Protein-rich diets greatly facilitate the production of corticotrophin in animals under stress. On the other hand, in animals not exposed to alarming stimuli, the concentration of protein in the food exerts no significant action upon the adrenal cortex, unless the protein concentration is so high ( 70 to $90 \%$ ) that the diet itself becomes a stressor". Again to quote the same author: "In connection with GAS it is of particular interest that muscular exercise causes especially marked adrenal enlargement in rats having insufficient stores of thiamine, and that exogenous administration of yeast prevents this hypertrophy".

"The adrenal lesions in thiamin deficient animals are generally accompanied by thymus involution and other GAS manifestations, and have hence been interpreted as part of this syndrome". This view received further support by histochemical studies.

Ascorbic acid deficiency causes especially pronounced adrenal lesions in the guinea-pig which is notoriously sensitive to scurvy. There is marked enlargement and hyperaemia of the cortex; at the same time haemorrhages are particularly common, especially along the cortico-medullary junction line.

It is difficult to pinpoint the connection between diet and leprosy or lepra reaction, but on the whole there is a correlation between the experimental findings above and the writer's experience of leprosy in India. Throughout that country malnutrition is widespread, but it has 
been found that leprosy is most common in areas which are subject to periodic famine following failure of the monsoons, or destruction of crops by floods, thus causing occasional severe starvation.

Physical exercise is important in the treatment of leprosy, but it must be backed by adequate nourishment. Yet' it is remarkable how leprosy patients, inured to hard physical work on a diet of little more than a scanty ration of rice or other cereal, along with some form of lentils and a few vegetables, make satisfactory improvement towards recovery.

On the other hand, rich feeding without sufficient exercise is positively harmful, as may be shown by one example typical of many known to the writer. The son of a rich Indian landowner, while his father was still alive, led a physically strenuous life as an engineer. His history showed that for years he had been infected with leprosy, but the symptoms had not been sufficient to attract his attention and lead to a diagnosis. On his father's death, however, he inherited wealth and took up a more sedentary selfindulgent life. Within six months the signs of leprosy appeared in an acute exacerbated form.

The commonest food deficiency among leprosy patients in India is that of vitamins, especially vitamin B; and yeast is found to be beneficial, especially in children. A common indigenous remedy is found in various forms of home-made beer.

4. Accompanying and Complicating Conditions. These have an important bearing on lepra reaction, but the effects they produce vary considerably according to the condition of the patient, the type and advancement of the leprosy infection, and the acuteness, chronicity and nature of the complicating disease.

Vaccination for smallpox in a leprosy institution is well known to give rise to lepra reaction in a number of the patients. So is influenza or any acute virus epidemic. Chronic conditions, such as ankylostomiasis and subclinical malaria or filaria, are often found in India to underlie persistent or relapsing lepra reaction. Those complications may be interpreted according to the GAS as affecting the adrenal cortex and causing lepra reaction through hypocorticoidaemia over a shorter or more prolonged period. In leprosy one of the most damaging accompanying conditions is mental distress induced by the social stigma associated with the disease, which often affects the patient and his reactions and involves loss of employment and worries regarding means of liveli hood.

Climatic changes such as excessive heat or cold are often responsible for inducing lepra reaction.

\section{Summary of Relationship of Leprosy to the GAS}

1. The General Adaptation Syndrome in its true stages of Alarm Reaction, Resistance and Exhaustion is evoked not as a uniform response to a single agent but as a response to many varied agents. 
In this it corresponds with and offers an explanation of the nature of lepra reaction which is also evoked by many varied agents.

2. GAS also explains the difference between ENL and acute exacerbation. In the former tolerance is gained during the stage of resistance; while in the latter, stressors too'strong or too prolonged lead to exhaustion.

3. Lepra reaction is represented as a result of hypocorticoidaemia. This hypothesis is supported by the fact that the oral administration of corticoids rapidly relieves, at least for a short period, the symptoms of the more severe form of lepra reaction.

4. The tissue changes described by SFI.YE as those elicited by the Alarm Reaction of GAS (blood concentration, deranged capillary and cell membrane permeability, and generalised tissue breakdown) correspond largely with those found in lepra reaction.

5. Out of many stressor agents mentioned by Selye four are chosen as particularly relevant to the causation of lepra reaction, viz. sulphur derivatives, muscular exercise, defective diet, and accompanying conditions.

6. The stressor effects of certain sulphur derivatives on experimental animals, and particularly on the adrenal cortex, are discussed, and it is suggested that similar effects may be caused by sulphones, thus accounting for ENL so frequently occurring in the beginning of treatment.

7. It is shown how graded muscular exercise in animals strengthens the adrenal cortex, and increases general adaptation to other stresses, while excessive exercise has an opposite effect through damage to the adrenal cortex.

8. Similarly in leprosy patients muscular training may be used to prevent and remedy lepra reaction. The adverse effects of excessive rest and sedentary habits are mentioned.

9. In the treatment of leprosy physical training must be supported by suitable diet. Both unbalanced diet and excessive indulgence are apt to give unfavourable results.

10. Acute and chronic accompanying diseases, and other unfavourable conditions such as mental distress and climatic changes may underlie lepra reaction, and their diagnosis and treatment are of importance.

\section{The Prevention of Lepra Reaction}

The milder form, ENL, when caused by excessive initial chemotherapy, can be prevented as a rule by grading the early doses according to the tolerance of the patient. When careful examination shows that the general resistance of the patient is low, sulphone treatment should be preceded by remedying the general condition. Accompanying diseases should be diagnosed and treated, and weakening conditions such as mental anxiety and deficient nutrition 
rectified as far as possible. This does not mean that sulphone or other specific treatment is to be completely withheld until all deficiencies have been entirely adjusted, but their presence should be kept in mind when grading the early dosage, and weak patients should be kept under frequent and careful inspection.

The importance of getting into, and remaining in 'training' by means of suitable, graded physical exercise backed by good nutrition has already been emphasised. The patient should be taught that he has to walk towards recovery on two limbs-physical training and chemotherapy, and that neglect of or interference with either will delay or imperil his ultimate cure. It has been repeatedly found by clinicians with wide experience of leprosy treatment that lepra reaction, especially in its severe $\mathrm{AE}$ form, is most common among educated 'better class' patients with sedentary habits, and comparatively rare among those whose livelihood is by the 'sweat of the brow'; also that, while defective nutrition is harmful, excessive indulgence in eating and drinking is even more likely to be accompanied by a tendency to lepra reaction. Explained in the light of the general Adaptation Syndrome, those 'in training' are strongly conditioned and adapted to resist the stresses of leprosy itself, of sulphone or other specific treatment, and of adverse complicating conditions. We may consider that in these patients the endocrine system, and particularly the adrenal cortex, is healthy and functioning strongly, and can enable them to stand up to intercurrent adverse circumstances.

\section{Treatment of Lepra Reaction}

The milder type of reaction (ENL), when induced by specific treatment with sulphone or other drugs, requires as a rule only temporary suspension or lowering of the dosage.

Acute exacerbation with inflammation, febrile symptoms, neuritis, and pain in the affected parts requires not only suspension of sulphones or other specific treatment but also bed-rest, laxatives, diaphoretics, alkalis and analgesics.

It is important to bear in mind the possibility of some underlying condition which must be diagnosed and treated if the symptoms continue; recourse should be had to some of the well-known forms of treatment of $\mathrm{AE}$, chief among which is the intravenous injection of antimony compounds (potassium antimony tartrate, fuadin, etc.). Only when these fail to bring the condition under control, or when symptoms are particularly acute or urgent (severe nerve pain, inflammatory eye conditions, for example), should corticoids be used. Also in a disease like leprosy where there is a tendency toward osteoporosis and neuropathy, these conditions are apt to be aggravated by large or long-continued dosage with corticoids or by their sudden suppression. 
Two methods of corticoid treatment of AE have been practiced in recent years.

1. Large doses of prednisone are given (10 to $20 \mathrm{mgm}$. daily) with gradual reduction of the dose as the symptoms subside. After this sulphone or other form of specific treatment is continued, its reaction-producing effects being countered with an adequate quantity of prednisone. The objection to this procedure is that fairly large quantities of corticoid have often to be continued over a long period, which implies the danger of producing atrophy of the adrenal cortex, with addiction to corticoids and production of the Cushing syndrome.

2. The other method of procedure, recommended by the writer, is to suspend all specific treatment until the general resistance of the patient has been built up. Begin with minimal doses of prednisone ( $1 \frac{1}{2}$ to $3 \mathrm{mgm}$. daily), and increase the amount slowly only if improvement fails to appear within 1 or 2 days. As soon as the AE has been brought under control, begin graded physical exercises. These may have at first to be of passive nature if the strength of the patient is much reduced or there is residual pain or stiffness. Certain parts may have at first to be kept at rest (such as an arm or leg by means of bandage, sling, cast or special footwear), but the patient should be encouraged as soon as possible to take active exercise, carefully regulated to his capacity. Further corticoid treatment should be held in reserve, and only given (in minimal doses) if there are signs of relapse of reaction. In the writer's experience it is possible in most cases to built up the physical strength of the patient till at last he gets 'into training'. Only when the resistance of the patient is restored should specific treatment be continued, beginning with carefully graded amounts. The greatest dangers in AE consists in the patient becoming accustomed to bed-rest, and habituated to corticoid medication.

A special note is necessary regarding the prevention and treatment of irido-cyclitis. In any form of lepra reaction the eyes should always be examined, and any sign of ocular pain or inflammation, sluggish, fixed or irregularly shaped pupil should be an indication for dilatation of the pupil with mydriatics.

\section{Further Study}

This paper suggests certain questions which require further study.

1. What are the effects of sulphones on the adrenal cortex of animals?

2. What are the effects of iodides on the adrenal cortex in animals? Iodides are well known to have a powerful effect in producing lepra reaction. Is this due to indirect action on the adrenal cortex via the thyroid? 
3. What is the effect of thyroxine administration in leprosy and in lepra reaction?

4. What is the effect of $\mathrm{NaCl}$ administration in lepra reaction?

5. Could $M$. leprae be successfully inoculated in animals after suppression or partial suppression of the adrenals?

\section{Summary}

1. It is sought to explain lepra reaction in its two forms of Erythema nodosum leprosum (ENL) and acute exacerbation (AE) by means of the General Adaptation Syndrome (GAS) of Selye.

2. Lepra reaction appears not as in allergy as a uniform response to one agent, but as a response to a large number of widely differing agents.

3. It is suggested that the difference between ENL and AE lies in success in producing tolerance to certain stressors in the former, and failure to do so in the latter.

4. An explanation is offered for the appearance of ENL at the beginning of sulphone treatment.

5. The effects of physical exercise, diet and complicating conditions are discussed in the light of the GAS.

6. The prevention and treatment of lepra reaction are discussed with special reference to physical training and cortisone therapy.

7. Certain questions are raised by this paper which require further investigation. 